\title{
Artificial Odor Map and Cluster Sensing by MIP Adsorbents
}

\author{
Masahiro Imahashi, Koichi Nakano, Kenshi Hayashi \\ Information Science and Electrical Engineering, Kyushu University, Fukuoka, Japan, \\ imahashi@o.ed.kyushu-u.ac.jp
}

\begin{abstract}
In this study, we focused on an appropriate classification of different odorants like biological odor clustering and constructed the odor map by sensor technology to achieve the odor discrimination. First, an sensor system that imitates the odor receptive mechanism of biological olfaction was developed. With some adsorbents, this system enables high detection and discrimination of odor by obtaining molecular parameters of odorants. Odor features can be extracted by measuring representative odor materials that belong to different glomeruli clusters. For the purposes, we developed nano-filter adsorbents, called as molecular imprinted polymer adsorbents (MIPAs), which have high molecular recognition ability. MIPAs enable the classification of odorants into belonging odor clusters. Finally, we measured the selectivity of MIPAs used poly (acrylic acid) (PAA) or peptide as MIP layer. MIPAs make it possible odor clustering and the construction of odor map in the biological olfaction.
\end{abstract}

Key words: odor map, molecular imprinted polymer, odor clustering, discrimination, molecular sieve

\section{Introduction}

Recently, there are urgent needs for sensing and digitization of odor information. However, sensors comparable to the ability of biological olfaction have still not reached a practical level. Qualitative odor measurement might be possible by imitating the recognition processes of biological olfaction.

Several specific olfactory receptors (ORs) corresponding to one odorant are activated when mammals smell odors $[1,2]$. Then, the active signals of ORs are separately transmitted to definitely located glomeruli on the surface of the olfactory bulb, which is the first terminal connected to ORs [3]. Then, the odor map integrated receptor signals is formed on glomeruli $[4,5,6]$. Thus, information of the molecular structure of odorants are transformed into map on the olfactory bulb.

Recently, it is found that neighboring glomeruli have similar molecular-receiving properties and mammals form the map composed of some clusters, e.g., carboxylic acids belong to cluster A $[2,7]$. Odorants that have similar molecular structures activate glomeruli that have similar characteristics and are classified into the same cluster in the biological olfaction process $[6,8]$. In fact, it is said that odor measurement can be realized by constructing the odor-cluster map. Hence, we developed the odor separating system (Fig. 1) to construct the artificial odor map classified by their odor-cluster attributes.

\section{Odor Separating System}

The odor separating system enables the separation and detection of odorants by the molecular sieve effect of some adsorbents, and classification of odorants by the construction of odor map. The system mainly consists of separating cells, where micro-ceramic heaters are embedded, and metal oxide (MOX) gas sensors. On the surface of heaters, some adsorbent materials with different properties are adhered. Therefore, odorants injected to separating cells are trapped to adsorbents with the different properties. Then, after heating up each heater to separately desorb odorants, MOX sensors measure separated odorants.

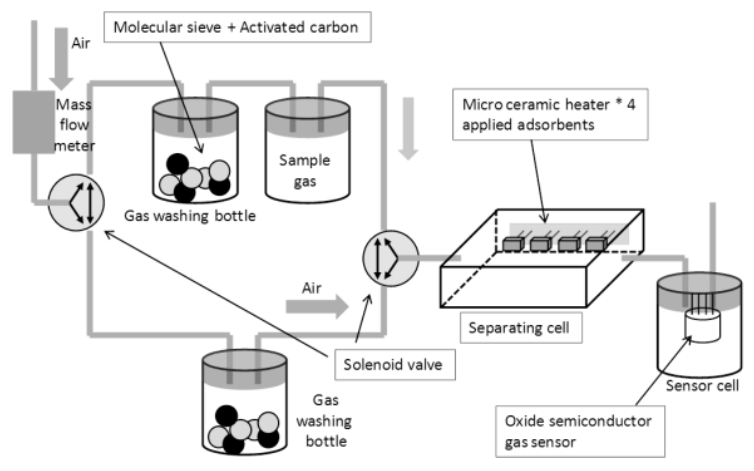

Fig. 1. Odor separating system

The specific adsorbent materials make it possible to measure molecular parameters of odorants and estimate clusters. In this study, we succeeded to estimate molecular size and polarity of odorants by the system applied 
carbon molecular sieves, and gaschromatographic column adsorbent materials [9]. In addition, we constructed artificial odor map based on measured molecular parameters Fig. 2 shows the artificial odor map based on molecular size and polarity of odorants. As shown in Fig. 2, the map is roughly classified according to each cluster. Consequently, the system can evaluate various odorants and maps into clusters.

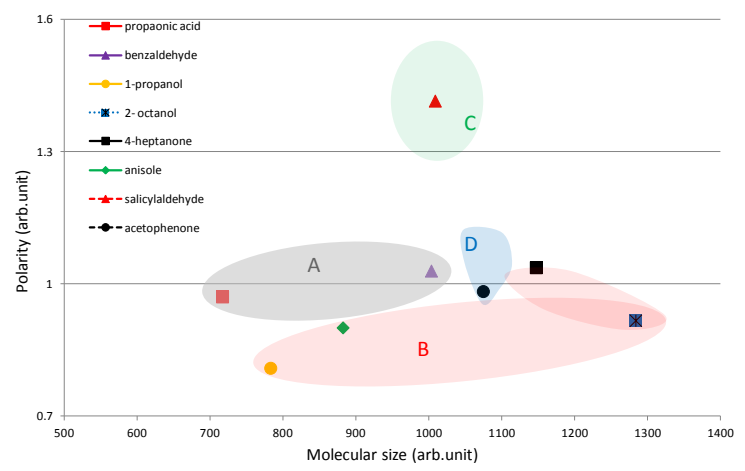

Fig. 2. Artificial odor map constructed by molecular parameters using the odor separating system

\section{Molecular Imprinted Polymer Adsorbent (MIPA)}

It is necessary to obtain sufficient odorant information about each cluster for the odor map. However, conventional adsorbents are inferior in terms of molecular recognition of odorants. In addition, because molecular structures of many odorants belonging to each cluster are mapped in the odor map, adsorbents which can discriminate the shape or functional groups of odorants are essential. Therefore, we developed MIPAs; adsorbents with molecular imprinted polymer (MIP) surface which have a specific binding site for template odorants belonging to odor cluster for embedding to the system. MIPAs have an odor molecular sieving effect and enable the determination of clusters.

MIPAs with modified surfaces by poly (acrylic acid) (PAA) layer maintained the shape of a template odorant is composed on a polydimethylsiloxane (PDMS) substrate. PDMS, whch is used as an adsorption layer of solid phase micro extraction (SPME), has high adsorption ability, therefore, it can become adsorption layer of MIPAs. Fig. 3 shows the layer structure of MIPAs. The purpose of this adsorbents is to generate desorbed odorant gases after heating up. Because the MIP layer is ultrathin and not used as adsorption layer, MIPAs can avoid unspecific adsorption.

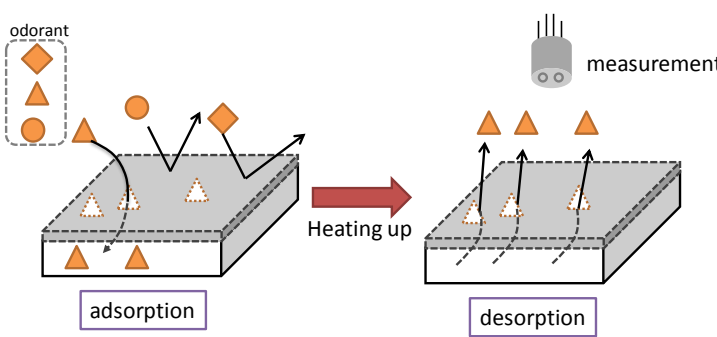

Figure 3: Adsorption and desorption of odorants

\section{Modification of MIP}

Fig. 2 outlines the surface modification process and imprinting process which includes assembly of PAA/template comlex on a $\mathrm{TiO}_{2}$ monolayer [10]. A typical preparating procedure of MIPAs is as follows. First, the surface of PDMS was plasma-treated in order to surfaceactivate. Second, the hydrophilized PDMS surface was immersed in a $100 \mathrm{mM}$ solution of titanium tetra-butoxide in 1:1 (v/v) toluene/ethanol for $20 \mathrm{~min}$, and rinsed in ethanol. Third, it was immersed in a $10 \mathrm{mM}$ ethanolic solution of a mixture of PAA, target odorant, and hydrochloric acid $(10,5$, and $50 \mathrm{mM}$ each) for 30 minutes, and then dried with $\mathrm{N}_{2}$ gas flushing after rinsed in ethanol. Finally, the fabricated MIPA was heated at 100 Celsius for more than 1 hour in order to desorb target odorant from the MIP matrix.
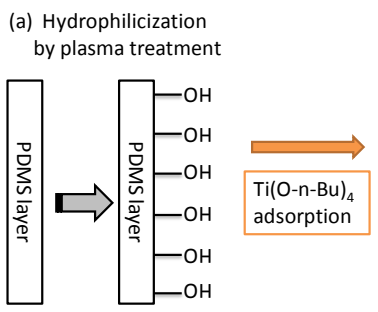

(b) Adsorption of Ti laye
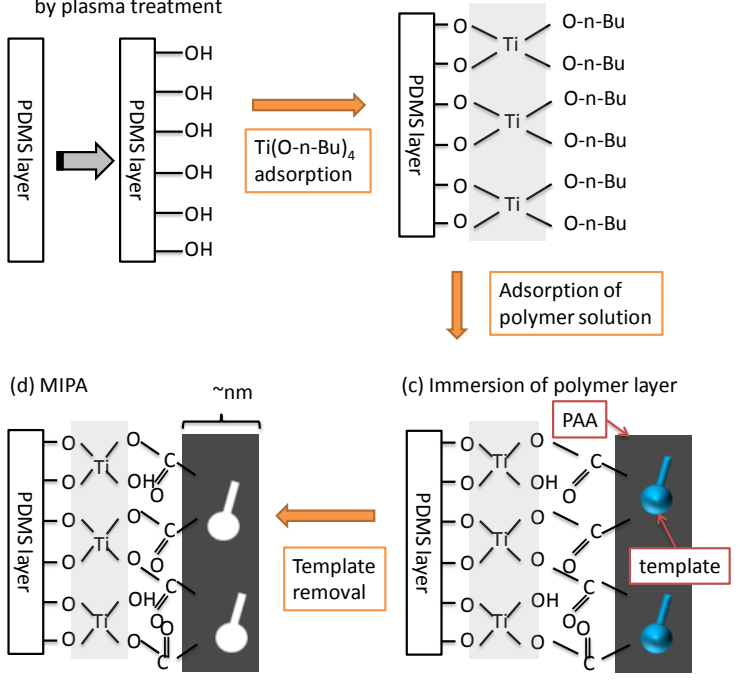

Fig. 4. Molecular imprinting via surface modification with PAA/template complex

\section{Measurement of Adsorption Amount of Gases}

In this study, the selectivity of MIPAs was evaluated by comparing between the adsorption amounts of single odorant or mixed odor to MIPAs and the ones to PDMS without MIP film (native-PDMS). Fig. 5 indicates the measuring procedure of the selectivity of 
odorants to MIPAs using gas chromatography mass spectrometry (GC/MS) and SPME method. First, Both a MIPA and native-PDMS were set in glass container, which packed saturated single odor or mixed odor, for $20 \mathrm{~min}$. Then, odorants trapped to adsorbents were sampled using SPME after heating up each adsorbents. Finally, the adsorption property of MIPA were calculated by comparing each adsorption response obtained with GC/MS.

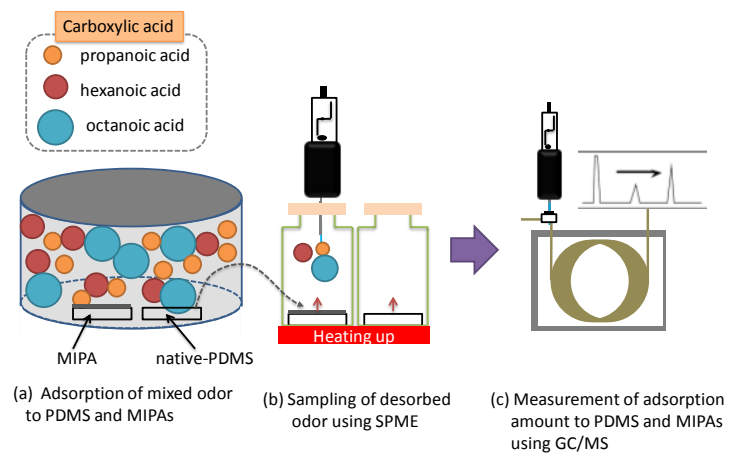

Fig. 5. Measurement of selectivity of odorants to MIPAs and PDMS

\section{Results and Discussion}

We measured the adsorption property of MIPAs with a site for three kinds of carboxylic acids to single odor or mixed odor of them to confirm the selectivity of MIPAs. The selectivity results in single odor and the mixed odor are shown in Fig. 6 (a) and (b), respectively. The vertical axis in Fig. 4 was normalized by the adsorption amounts of MIPAs to native-PDMS.

Fig. 6 (a) indicates that propanoic-acid MIPA could selectively trap a template odorant and reject odorants with larger molecular sizes. In addition, other MIPAs most selectively are adsorbed to template odorant and has a molecular sieve effect, because smaller odorants could pass through the MIP filter and adsorb into PDMS, but odorants with a longer molecular size could not do. These results suggested that MIP with molecular sieve effect was formed on PDMS, and MIPAs successfully adsorbed only odorants which pass through imprinted template site.

In the case of mixed odor (Fig. 6(b)), all MIPAs selectively adsorbed to template odorant, and could catch more odorants with a low proportion of the saturated mixed odor, but adsorbed to less odorants with a smaller size than template odorant. It is thought that MIPAs represents the specific adsorption to template odor in the presence of the various kinds of chemicals.

\section{Peptide MIPA}

PAAs used as filter materials of MIPAs cannot form a specific site for all odorants. Therefore,
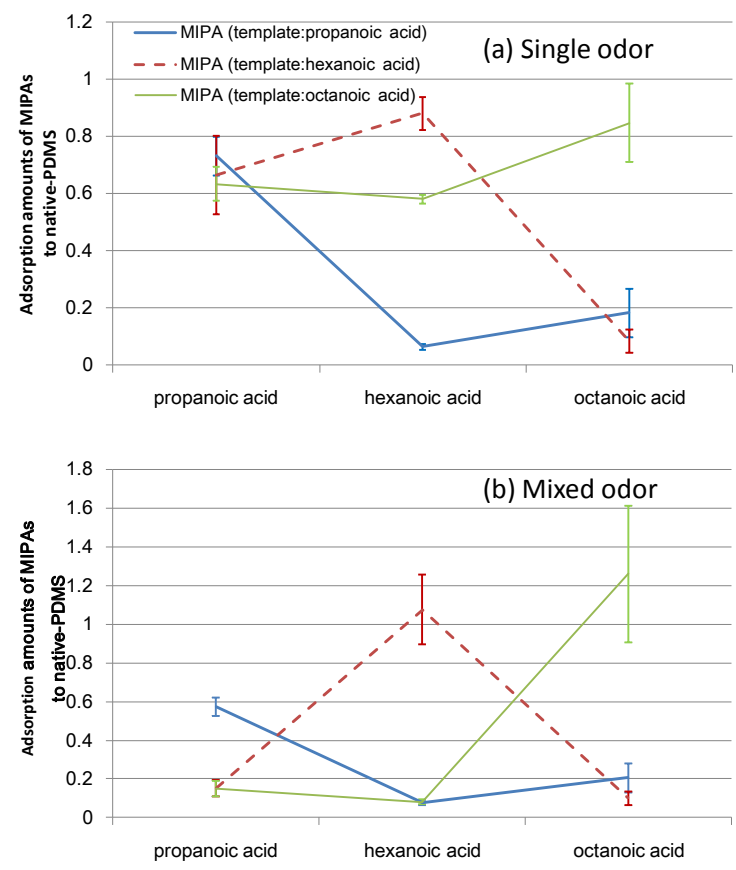

Fig. 6. Selectivity of MIPAs with a site of carboxylic acid in the (a) single odor or (b) mixed odor

as a new approach, we developed peptide MIPA replaced filter materials to peptide. Here, ORs consist of proteins and catch every odorants by the combination of different peptides, e.g., olfactory receptor proteins (large peptide polymers) selectively traps odor molecules. Therefore, it can be said that peptide MIPAs are extensive adsorbents and make it possible the molecular sieve of odorants close to ORs.

In this study, aspartic acids were adopted as constituent amino acid of peptide. The procedure of peptide MIPAs is as follows. After $\mathrm{Ti}$ layer was immersed on PDMS whose surface was hydrophilized by plasma treatment, it was immersed in a ethanolic solution of a mixture of aspartic acid and hydrochloric acid $(10 \mathrm{mM}$ and $0.1 \mathrm{M}$ each) for 30 minutes, and then a peptide layer was formed after 30 minutes from the injection of $90 \mu \mathrm{mol}$ amide condensation agent. Finally, PDMS modified with the peptide layer was immersed in a ethanolic solution of $10 \mathrm{mM}$ template odorant (hexanoic acid or octanoic acid) for 1 day.

We confirmed the selectivity of peptide MIPAs with a site for template odorant to mixed odor of three kinds of carboxylic acids by comparing between the adsorption amounts to MIPAs and the ones to native-PDMS using GC/MS and SPME. The selectivity results of peptide MIPAs are shown in Fig. 7. Fig. 7 indicates that peptide MIPAs can not only be adsorbed to most template odorant, but also pass through other 
odorants with a smaller size than template odorant in comparison with PDMS.

PAA-MIPAs certainly has a higher filter ability than peptide MIPAs because of the firmness of the polymer matrix, but catch only template odorant in the mixed odor. On the other hand, peptide MIPAs maintain the molecular sieve effect even in the mixed odor. Therefore, it is said that peptide MIPAs are efficient in the adsorption of odorants in the mixed odor. In fact, clusters can be determined by measuring desorption of odorants from adsorbents, embedding MIPAs in the odor separating system.

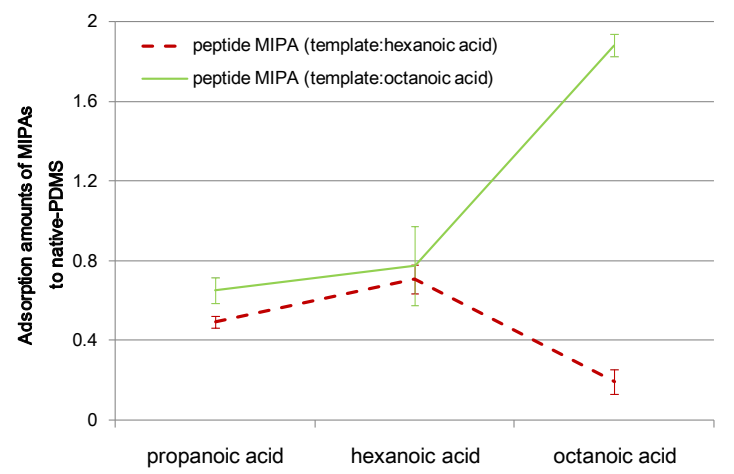

Fig. 7. Selectivity of peptide MIPAs with a site of carboxylic acid for mixed odors

\section{Conclusion}

In this study, we developed PAA and peptide MIPAs with an advanced molecular recognition ability. They are especially effective in the adsorption of odorants with a quite low proportion of mixed odor. The adoption of peptides as MIP materials lead to the extensive application, e.g., MIPAs with wide variety of molecular structures can be developed.

In addition, the odor separating system embedded MIPAs makes it possible to construct the odor map in the biological olfactory. Fig. 8 shows an example of the image map (cluster A) constructed from sensor responses of MIPAs representing each cluster. As shown by Fig. 8, the map simultaneously activated some clusters such like the biological odor map. Indeed, it is thought that the map is the internal expression of biological olfactory sense.

\section{References}

[1] K. Mori, Y. Yoshihara, Molecular recognition and olfactory processing in the mammalian olfactory system, Prog. Neurobiol. 45, 585-619 (1995) ; doi: 10.1016/0301-0082(94)00058-P

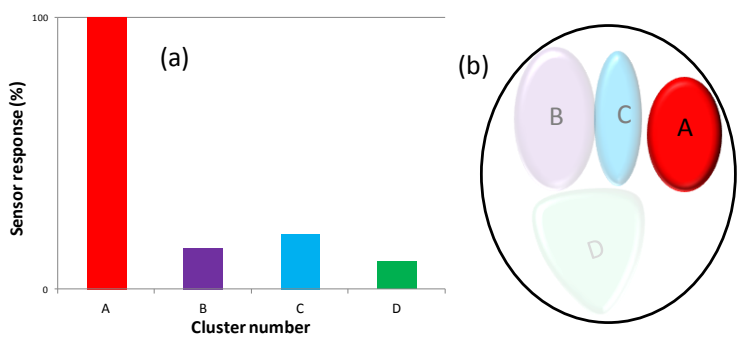

Fig. 8: The odor map model

(a) Sensor responses of MIP adsorbents

(b) the odor map based on sensor responses

[2] K. Mori, Y.K. Takahashi, K. Garashi, M. Yamaguchi, Maps of odorant molecular features in the mammalian olfactory bulb, Phys. Rev. 8, 409-433 (2006); doi: 10.1152/physrev. 00021.2005

[3] L. Buck, Olfactory receptors and odor coding in mammals, NUTR. REV. 62, 184-188 (2004); doi: 10.1301/nr.2004.nov.S184-S188

[4] C. Natale, E. Martinelli, R. Paolesse, A. D'Amico, D. Filippini, I. Lundström, An experimental biomimetic platform for artificial olfaction, PLOS ONE. 3 (2008) e3139; doi :10.1371/journal.pone.0003139

[5] C. Galizia, S. Sachse, A. Rappert, R. Menzel, The glomerular code for odor representation is species specific in the honeybee Apis mellifera, Nat. Neurosci. 2, 473-478 (1999) ; doi: 10.1016/S0022-1910(99)00194-8

[6] T. Bozza, A. Vassalli, S. Fuss, J.J. Zhang, B. Weiland, R. Pacifico, P. Feinsteia, P. Mombaerts, Mapping of class I and class II odorant receptors to glomerular domains by two distinct types of olfactory sensory neurons in the mouse, Neuron 61, 220-233 (2009) ; doi: 10.1016/j.neuron.2008.11.010

[7] H. Matsumoto, K. Kobayakawa, R. Kobayakawa, T. Tashiro, K Mori, H. Sakano, K. Mori, Spatial arrangement of glomerular molecular-feature clusters in the odorant-receptor class domains of the mouse olfactory bulb, J. Neurophysiol. 103, 3490-3500 (2010) ; doi:10.1152/jn.00035.2010

[8] B. Johnson, M. Leon, Chemotopic Odorant Coding in a Mammalian Olfactory System, $\mathrm{J}$. Comp. Neurol. 503, 1-34 (2007) ; doi: $10.1002 /$ cne. 21396

[9] M. Imahashi and K. Hayashi, Odor clustering and discrimination using an odor separating system, Sensor and Actuators B, in press (2011) ; doi: 10.1016/j.snb.2012.03.041

[10] D. Yang, M. Ju, A. Maeda, K. Hayashi, K. Toko, S. Lee, T. Kunitake, Design of highly efficient receptor sites by combination of cyclodextrin units and molecular cavity in $\mathrm{TiO} 2$ ultrathin layer, Biosens Bioelectron. 22, 388-92 (2006) ; doi: 10.1016/j.bios.2006.06.013 